\title{
Efficient algorithms for Hirshfeld-I charges
}

\author{
Kati Finzel, Angel Martin Pendas and Evelio Francisco
}

\section{Linköping University Post Print}

\section{Tweet}

N.B.: When citing this work, cite the original article.

Original Publication:

Kati Finzel, Angel Martin Pendas and Evelio Francisco, Efficient algorithms for Hirshfeld-I charges, 2015, Journal of Chemical Physics, (143), 8, 084115.

http://dx.doi.org/10.1063/1.4929469

Copyright: American Institute of Physics (AIP)

http://www.aip.org/

Postprint available at: Linköping University Electronic Press

http://urn.kb.se/resolve?urn=urn:nbn:se:liu:diva-122068 


\section{AP $\mid \begin{aligned} & \text { The Journal of } \\ & \text { Chemical Physics }\end{aligned}$}

\section{Efficient algorithms for Hirshfeld-I charges}

Kati Finzel, Ángel Martín Pendás, and Evelio Francisco

Citation: The Journal of Chemical Physics 143, 084115 (2015); doi: 10.1063/1.4929469

View online: http://dx.doi.org/10.1063/1.4929469

View Table of Contents: http://scitation.aip.org/content/aip/journal/jcp/143/8?ver=pdfcov

Published by the AIP Publishing

\section{Articles you may be interested in}

Deriving the Hirshfeld partitioning using distance metrics

J. Chem. Phys. 141, 094103 (2014); 10.1063/1.4894228

An efficient polyenergetic SART (pSART) reconstruction algorithm for quantitative myocardial CT perfusion Med. Phys. 41, 021911 (2014); 10.1118/1.4863481

A Hirshfeld-I interpretation of the charge distribution, dipole and quadrupole moments of the halogenated acetylenes $\mathrm{FCCH}, \mathrm{CICCH}, \mathrm{BrCCH}$, and $\mathrm{ICCH}$

J. Chem. Phys. 133, 214103 (2010); 10.1063/1.3511784

Singular value decomposition based computationally efficient algorithm for rapid dynamic near-infrared diffuse optical tomography

Med. Phys. 36, 5559 (2009); 10.1118/1.3261029

Theoretical Level Structure And Decay Dynamics For the Be-like Ti lon AIP Conf. Proc. 748, 93 (2005); 10.1063/1.1896480

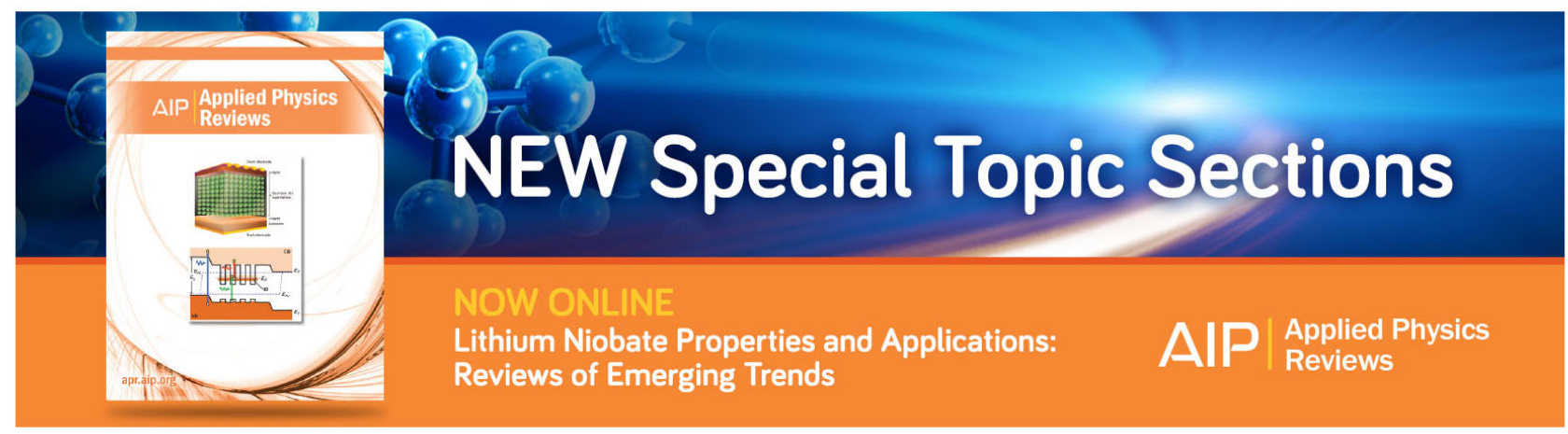




\title{
Efficient algorithms for Hirshfeld-I charges
}

\author{
Kati Finzel, ${ }^{1, a)}$ Ángel Martín Pendás, ${ }^{2}$ and Evelio Francisco ${ }^{2}$ \\ ${ }^{1}$ Linköpings University, Department of Physics (IFM), 58183 Linköping, Sweden \\ ${ }^{2}$ Dpto de Química Física y Analítica, Facultad de Química, Universidad de Oviedo, 33006-Oviedo, Spain
}

(Received 14 April 2015; accepted 12 August 2015; published online 26 August 2015)

\begin{abstract}
A new viewpoint on iterative Hirshfeld charges is presented, whereby the atomic populations obtained from such a scheme are interpreted as such populations which reproduce themselves. This viewpoint yields a self-consistent requirement for the Hirshfeld-I populations rather than being understood as the result of an iterative procedure. Based on this self-consistent requirement, much faster algorithms for Hirshfeld-I charges have been developed. In addition, new atomic reference densities for the Hirshfeld-I procedure are presented. The proposed reference densities are N-representable, display proper atomic shell structure and can be computed for any charged species. (C) 2015 AIP Publishing LLC. [http://dx.doi.org/10.1063/1.4929469]
\end{abstract}

\section{INTRODUCTION}

The Hirshfeld atoms ${ }^{1}$ are a prominent partitioning scheme in quantum mechanics. Recently, it has been extended to an iterative version. ${ }^{2}$ One of the major benefits of that iterative scheme $^{2}$ compared to the original Hirshfeld method ${ }^{1}$ is the independence of the atomic reference state (whether the promolecule is formed by charged or uncharged fragments). That benefit has made the Hirshfeld-I partitioning scheme gain increasing interest in chemical bonding analysis. ${ }^{3,4}$ It also shows promise in force-field development. ${ }^{5}$

This study provides a new and simple viewpoint on atoms obtained via the iterative Hirshfeld method (Hirshfeld-I), ${ }^{2}$ from which efficient algorithms for calculating Hirshfeld-I charges can be constructed. The proposed algorithms are build on the ideas of Bultinck et al., ${ }^{6}$ but their logical extension has not been published yet.

The Hirshfeld-I procedure uses atomic reference densities obtained from self-consistent calculations. This causes problems when highly charged anions are needed as input reference data, since no doubly charged anion is stable..$^{5}$ To circumvent this problem, an extended Hirshfeld-I procedure has been proposed, ${ }^{5}$ constructing atomic reference densities of charged species by scaling the shape function of the corresponding neutral atoms. Especially for solids, the attempt has been taken in the direction of calculating reference atoms in a periodic box and neglecting the long range part of the anionic electron densities. ${ }^{4}$ Both attempts aim in a certain way to adjust the size of the anionic electron density. This study provides another scheme of how to construct atomic reference densities, that can easily be obtained for any charged or uncharged species. The proposed reference densities are N-representable, exhibiting proper shell structure and their size can be adjusted by one meaningful parameter.

The paper is organized as follows. In Sec. II A, the current version of Hirshfeld-I is repeated. Hereafter, the improved Hirshfeld-I scheme is presented. That section contains the

\footnotetext{
a)kati.finzel@cpfs.mpg.de; On leave from Max Planck Institute for Chemical Physics of Solids, Nöthnitzer Str. 40, 01187 Dresden, Germany.
}

main key ideas on which the methodological development is based, followed by some technical details and the extensive testing of the new proposed algorithms. In the last part, the new reference densities are presented.

\section{RESULTS AND DISCUSSION}

\section{A. The iterative Hirshfeld method}

The iterative Hirshfeld (Hirshfeld-I) method has been proposed by Bultinck et al. ${ }^{2}$ in order to correct for certain shortcomings of the original Hirshfeld method, ${ }^{1}$ such as the occurrence of almost zero charges, ${ }^{7,8}$ problems when extending the scheme to charged molecules, ${ }^{2}$ and the bias due to the atomic reference state. ${ }^{9,10}$

In the original Hirshfeld partitioning scheme, the weighting function $w_{A}(\vec{r})$ for atom $A$ in a molecule formed by $M$ atoms is constructed from the spherical atomic density $\rho_{A}^{0}(\vec{r})$ of the free atom $A$,

$$
w_{A}(\vec{r})=\frac{\rho_{A}^{0}(\vec{r})}{\sum_{A}^{M} \rho_{A}^{0}(\vec{r})} .
$$

Clearly, $w_{A}(\vec{r})$ depends on whether the molecule is thought of being composed of neutral or charged fragments. The electronic population $N_{A}$ of fragment $A$ in the molecule,

$$
N_{A}=\int \rho_{A}(\vec{r}) \mathrm{d} \vec{r}=\int w_{A}(\vec{r}) \rho(\vec{r}) \mathrm{d} \vec{r},
$$

of course also depends on the chosen atomic reference state. The major benefit of the iterative Hirshfeld scheme is to avoid that bias. In the Hirshfeld-I procedure, the weighting function $w_{A}^{i}(\vec{r})$ during iteration step $i$ is determined by the population $N_{A}^{i-1}$ of the previous step,

$$
w_{A}^{i}\left(N_{A}^{i-1} ; \vec{r}\right)=\frac{\rho_{A}^{i-1}\left(N_{A}^{i-1} ; \vec{r}\right)}{\sum_{A}^{M} \rho_{A}^{i-1}\left(N_{A}^{i-1} ; \vec{r}\right)},
$$

giving rise to the new charge $N_{A}^{i}$,

$$
N_{A}^{i}=\int w_{A}^{i}\left(N_{A}^{i-1} ; \vec{r}\right) \rho(\vec{r}) \mathrm{d} \vec{r} .
$$


This process is repeated until the absolute difference between the electron populations for two consecutive steps is below a given threshold for all atoms in the molecule. Since the electron populations $N_{A}$ are usually fractional numbers, the atomic densities colorgreen in each step are obtained by using a finite difference approach for the Fukui function, ${ }^{11}$

$$
\begin{aligned}
& \rho_{A}^{i}\left(N_{A} ; \vec{r}\right)=\left[u_{A}-N_{A}\right] \rho_{A}^{l_{A}}(\vec{r})+\left[N_{A}-l_{A}\right] \rho_{A}^{u_{A}(\vec{r})} \\
& =x_{A}\left[\rho_{A}^{l_{A}}(\vec{r})-\rho_{A}^{u_{A}}(\vec{r})\right]+\rho_{A}^{u_{A}}(\vec{r}), \\
& 0 \leq x_{A} \leq 1 \text {, }
\end{aligned}
$$

where $l_{A}=\operatorname{int}\left(N_{A}\right)$ and $u_{A}=\operatorname{int}\left(N_{A}\right)+1=l_{A}+1$ are the lower and upper integers to $N_{A}, x_{A}=u_{A}-N_{A}$, and $\rho_{A}^{l_{A}}(\vec{r})$ and $\rho_{A}^{u_{A}}(\vec{r})$ are promolecular atomic densities integrating to $l_{A}$ and $u_{A}$, respectively.

\section{B. The improved Hirshfeld-I method}

This section presents a new viewpoint on Hirshfeld-I charges, giving rise to the development of new efficient algorithms.

At the end of a successful iterative Hirshfeld procedure, the electronic population of fragment $A$ is given by

$$
N_{A}^{\text {end }}=\int w_{A}^{\text {end }}\left(N_{A}^{\text {end }} ; \vec{r}\right) \rho(\vec{r}) \mathrm{d} \vec{r}=f\left(N_{A}^{\text {end }}\right) .
$$

The weighting function $w_{A}\left(N_{A} ; \vec{r}\right)$ depends on $N_{A}$; therefore, the electron populations obtained from a converged Hirshfeld-I procedure can be interpreted as such populations which reproduce themselves. In the following, the suffix "end" is dropped since the self-consistent populations $N_{A}$ are seen as a requirement rather than the solution of an iterative procedure. The above analytic expression for Hirshfeld-I populations offers a straightforward route for obtaining the solutions to Eq. (7) considerably faster and more efficiently than the simple iterated scheme described in Sec. II A.

Let us consider for simplicity, a molecule $\left[\mathrm{A}_{a} \mathrm{~B}_{b}\right]^{q}$ formed by only two types of atoms A and B ( $q$ is the total charge, for a neutral molecule $q=0$ ), with all the atoms of type A (B) having the electron population $N_{A}\left(N_{B}\right)$. The generalization for an arbitrary molecule $\left[A_{a} B_{b} C_{c} \cdots\right]^{q}$ is given in the Appendix. The electron density of every atom of type $\mathrm{A}$ is given by Eq. (6), with an equivalent definition for atoms of type B. It should be noted that $N_{A}$ determines $l_{A}, u_{A}$, and $x_{A}$. Similarly, if one knows $l_{A}\left(\right.$ or $\left.u_{A}\right), x_{A}$ determines $N_{A}$. In terms of $x \equiv x_{A}$, Eq. (7) can be written as

$$
F(x)=\int w_{A}(x ; \vec{r}) \rho(\vec{r}) d \vec{r}-N_{A}(x)=0 .
$$

Writing each atomic density in form $(6), w_{A}(x ; \vec{r})$ may always be expressed as

$$
w_{A}(x ; \vec{r})=\frac{a(\vec{r}) x+b(\vec{r})}{c(\vec{r}) x+d(\vec{r})} .
$$

For instance, in $\mathrm{LiH}=\mathrm{AB}\left(x_{\mathrm{B}}=1-x_{\mathrm{A}}, l_{\mathrm{A}}=2, l_{\mathrm{B}}=1\right)$,

$$
\begin{aligned}
& a(\vec{r})=\rho^{\mathrm{Li}^{+}}(\vec{r})-\rho^{\mathrm{Li}^{0}}(\vec{r}), \\
& b(\vec{r})=\rho^{\mathrm{Li}^{0}}(\vec{r}), \\
& c(\vec{r})=\rho^{\mathrm{Li}^{+}}(\vec{r})-\rho^{\mathrm{Li}^{0}}(\vec{r})-\rho^{\mathrm{H}^{0}}(\vec{r})+\rho^{\mathrm{H}^{-}}(\vec{r}), \\
& d(\vec{r})=\rho^{\mathrm{Li}^{0}(\vec{r})+\rho^{\mathrm{H}^{0}}(\vec{r}) .}
\end{aligned}
$$

In the following, the spatial dependence will be suppressed for notational compactness. The values $x=0$ and $x=1$ give $w_{A}=\rho^{\mathrm{Li}^{0}} /\left[\rho^{\mathrm{Li}^{0}}+\rho^{\mathrm{H}^{0}}\right]$ and $w_{A}=\rho^{\mathrm{Li}^{+}} /\left[\rho^{\mathrm{Li}^{+}}+\rho^{\mathrm{H}^{-}}\right]$, respectively, that correspond to take the neutral atoms $\mathrm{Li}^{0}$ and $\mathrm{H}^{0}$ or the ions $\mathrm{Li}^{+}$and $\mathrm{H}^{-}$to build up the starting atomic promolecular densities. Analogous expressions of $a, b, c$, and $d$ can be derived for any $\left[A_{a} B_{b}\right]^{q}$ molecule.

Equation (8) can be solved using different strategies, all of them requiring the Taylor expansion of $w_{A}(x)$ about a point $x=x_{n}, w_{A}(x) \approx \sum_{i} w_{A}^{i}\left(x-x_{n}\right)^{i}$, where $w_{A}^{0}=w_{A}\left(x_{n}\right)$ and

$$
\begin{aligned}
w_{A}^{i} & =\frac{1}{i !}\left[\frac{d^{i} w_{A}(x)}{d x^{i}}\right]_{x=x_{n}} \\
& =\frac{(-1)^{i-1}(a d-b c) c^{i-1}}{\left(c x_{n}+d\right)^{i+1}}, \quad i \geq 1 .
\end{aligned}
$$

The successive derivatives of $F(x)$ at the point $x_{n}$ are

$$
\begin{aligned}
F^{\prime}\left(x_{n}\right) & =\int w_{A}^{\prime}\left(x_{n}\right) \rho(\vec{r}) d \vec{r}+1, \\
F^{\prime \prime}\left(x_{n}\right) & =\int w_{A}^{\prime \prime}\left(x_{n}\right) \rho(\vec{r}) d \vec{r}, \\
& \vdots \\
F^{i}\left(x_{n}\right) & =\int w_{A}^{i}\left(x_{n}\right) \rho(\vec{r}) d \vec{r} .
\end{aligned}
$$

Truncating the expansion of $w_{A}(x)$ at $i_{\max }=1$, Eq. (8) becomes to $F(x) \approx F\left(x_{n}\right)+F^{\prime}\left(x_{n}\right)\left(x-x_{n}\right)=0$. Solving for $x$, we have

$$
x_{n+1}=x_{n}-\frac{F\left(x_{n}\right)}{F^{\prime}\left(x_{n}\right)}=x_{n}+h_{n},
$$

which is the classical Newton method.

The iterative method known as Householders method consists of a sequence of iterations,

$$
x_{n+1}=x_{n}+d \frac{(1 / F)^{(d-1)}\left(x_{n}\right)}{(1 / F)^{(d)}\left(x_{n}\right)},
$$

beginning with an initial guess $x_{0}$. The Newton method corresponds to the 1st-order $(d=1)$ Householders method (H1). For $d=2(\operatorname{method} \mathrm{H} 2)$ and $d=3(\operatorname{method} \mathrm{H} 3)$, Eq. (19) may also be written, respectively, as

$$
x_{n+1}=x_{n}+\frac{h_{n}}{1+\frac{1}{2}\left[F^{\prime \prime}\left(x_{n}\right) / F^{\prime}\left(x_{n}\right)\right] h_{n}}
$$

and

$$
x_{n+1}=x_{n}+h_{n} \frac{1+\frac{1}{2}\left[F^{\prime \prime}\left(x_{n}\right) / F^{\prime}\left(x_{n}\right)\right] h_{n}}{1+\left[F^{\prime \prime}\left(x_{n}\right) / F^{\prime}\left(x_{n}\right)\right] h_{n}+\frac{1}{6}\left[F^{\prime \prime \prime}\left(x_{n}\right) / F^{\prime}\left(x_{n}\right)\right] h_{n}^{2}} .
$$


Finally, in the polynomial method (P), a 2nd-order expansion is first used for $w_{A}$ in Eq. (8),

$$
\begin{aligned}
F\left(x_{n}\right) \approx & \int\left[w_{A}^{0}+w_{A}^{\prime}\left(x_{n+1}-x_{n}\right)\right. \\
& \left.+w_{A}^{\prime \prime}\left(x_{n+1}-x_{n}\right)^{2}\right] \rho(\vec{r}) d \vec{r}-u_{A}+x_{n}=0 .
\end{aligned}
$$

Performing the integrations $\alpha=\int w_{A}^{0} \rho(\vec{r}) d \vec{r}, \beta=\int w_{A}^{\prime} \rho(\vec{r})$ $d \vec{r}$, and $\gamma=\int w_{A}^{\prime \prime} \rho(\vec{r}) d \vec{r}$ and reorganizing, one obtains $A x_{n+1}^{2}$ $+B x_{n+1}+C=0$, where $A=\gamma, B=\beta-2 \gamma x_{n}+1$, and $C$ $=\alpha-\beta x_{n}+\gamma x_{n}^{2}-u_{A}$. Solving this equation for $x_{n+1}$ (taking the solution $0<x_{n+1}<1$ ), setting $x_{n} \leftarrow x_{n+1}$, recomputing $A$, $B, C$, and so on, the process is iterated until $x_{n+1} \approx x_{n}$.

\section{Computational details and practical considerations}

The electron number distribution functions $(\mathrm{EDFs})^{12-14}$ of the second-row hydrides $\mathrm{AH}_{n}(\mathrm{~A}=\mathrm{Li}, \mathrm{Be}, \mathrm{B}, \mathrm{C}, \mathrm{N}, \mathrm{O}, \mathrm{F})$ computed with Hirshfeld-I atomic densities have been recently compared with those obtained from traditional (i.e., noniterative) Hirshfeld atoms. ${ }^{1}$ The above mentioned hydrides form a suitable test set probing the performance of the new iterative procedures, described in Section II B, compared to the simple fixed-point procedure of the standard HirshfeldI method. The GAMESS code $^{15}$ has been used to generate complete active space (CAS) SCF wave functions (CAS $[n, m]$, $n$ active electrons and $m$ active orbitals) with the standard 6$311 \mathrm{G}(\mathrm{d}, \mathrm{p})$ basis sets for the hydrides. The CAS descriptions used are CAS $[4,6]$ for $\mathrm{LiH}, \mathrm{CAS}[6,7]$ for $\mathrm{BeH}_{2}, \mathrm{CAS}[8,8]$ for $\mathrm{BH}_{3}$, and $\mathrm{CAS}[10,9]$ for $\mathrm{CH}_{4}, \mathrm{NH}_{3}, \mathrm{H}_{2} \mathrm{O}$, and HF. The reference atomic densities in the ground electronic states, obtained at the Hartree-Fock level and using also 6-311G(d,p) basis sets, were spherically averaged before being used in the iterative procedure. All the numerical integrations were performed with our domestic promolden code ${ }^{16}$ with an angular LebedevLaikov grid of 3890 points, and a radial grid of 200 points with the $r$-mapping procedure described in Ref. 17.

The iterative processes described in Section II B require for their implementations the lower $\left(l_{A}\right)$ or upper $\left(u_{A}=l_{A}+1\right)$ integer to the number of electrons, $N_{A}$, as well as the starting value of the interpolation parameter, $x_{A}$. From Eq. (6), starting with $x_{A}=0$ means that the initial guess for $N_{A}$ is $u_{A}$, while $x_{A}=1$ implies that initially $N_{A}=l_{A}$. The choice of $l_{A}$ or $u_{A}$ is not always trivial. For instance, the total charge of the $\mathrm{Li}$ fragment in the $\mathrm{LiH}$ molecule is clearly between 0 and +1 , $0<q_{A}<+1$, so there is no doubt in this case that $l_{A}=2$ $\equiv \mathrm{Li}^{+}, u_{A}=3 \equiv \mathrm{Li}^{0}, l_{B}=1 \equiv \mathrm{H}^{0}$, and $u_{B}=2 \equiv \mathrm{H}^{-}$. In $\mathrm{BeH}_{2}$, if $+1<q_{A}<+2$, one has $l_{A}=2 \equiv \mathrm{Be}^{2+}, u_{A}=3 \equiv \mathrm{Be}^{1+}, l_{B}$ $=1 \equiv \mathrm{H}^{0}$, and $u_{B}=2 \equiv \mathrm{H}^{-}$. However, if $0<q_{A}<+1$, one has $l_{A}=3 \equiv \mathrm{Be}^{+}, u_{A}=4 \equiv \mathrm{Be}^{0}$, and the same values of $l_{B}$ and $u_{B}$, so that $x_{B}$ as a function of $x_{A}$ is different. An analogous ambiguity exists in $\mathrm{BH}_{3}, \mathrm{CH}_{4}, \mathrm{NH}_{3}$, and $\mathrm{OH}_{2}$. Fortunately, a wrong initial guess for $l_{A}$ or $u_{A}$ is commonly detected after the first iteration step, since it provides an intermediate solution $x_{A}$ outside the defined value range $\left(x_{A} \neq[0,1]\right)$, which renders the readjustment of the initial guess fast and straightforward. For the proper initial guess, the procedure usually converges within 2-5 iterations.

\section{Comparison of algorithms}

We collect in Table I the results obtained for the $q_{A}$ values of the $\mathrm{AH}_{n}(\mathrm{~A}=\mathrm{Li}, \mathrm{Be}, \mathrm{B}, \mathrm{C}, \mathrm{N}, \mathrm{O}, \mathrm{F})$ hydrides. The starting value for $x_{A}$ (Eq. (6)) was 0.5 in all the cases, and appropriate values for the lower integers to $N_{A}\left(l_{A}\right)$ are $\mathrm{Li}^{+}, \mathrm{Be}^{2+}, \mathrm{B}^{+}, \mathrm{C}^{0}$, $\mathrm{N}^{-}, \mathrm{O}^{0}$, and $\mathrm{F}^{0}$. The final converged $q_{A}$ in a given hydride is the same in the five methods. However, the number of cycles required to achieve a convergence of 0.00001 is $6-10$ greater in the standard (std) procedure than in any of the methods put forward in this article. On the other hand, the difference between the H1, H2, H3, and P methods is not significant: $\mathrm{H} 1$ and $\mathrm{P}$ methods require the same number of cycles in all the cases, and $\mathrm{H} 2$ and $\mathrm{H} 3$ methods one cycle less than $\mathrm{H} 1$ and $\mathrm{P}$ methods in $\mathrm{BeH}_{2}, \mathrm{CH}_{4}, \mathrm{NH}_{3}$, and $\mathrm{H}_{2} \mathrm{Os}$, and the same number of cycles for other three hydrides. Since H2 method needs, besides $\boldsymbol{F}^{\prime}(\vec{x})$, the second derivatives $\boldsymbol{F}^{\prime \prime}(\vec{x})$, and H3 also the third ones $\boldsymbol{F}^{\prime \prime \prime}(\vec{x})$, the extra-time necessary to compute them does not compensates the reduction in the number of cycles in the event that this reduction actually occurs. In summary, assuming a similar implementation of all the numerical integrals required within Hirshfeld-I like electron population analyses, the simplest of the method proposed here (i.e., the $\mathrm{H} 1$ or Newton method) is about an order of magnitude faster than the standard iterative Hirshfeld scheme. The Newton method was applied to determine the Hirshfeld-I partitioning scheme for a wide test set, ranging from small molecules, such as $\mathrm{HF}^{+}, \mathrm{HCN}, \mathrm{CH}_{3} \mathrm{CLi}_{3}$, or $\mathrm{C}_{3} \mathrm{H}_{3}^{+}$, to larger systems, like the phenol dimer $\left(\mathrm{C}_{6} \mathrm{H}_{5} \mathrm{OH} \cdots \mathrm{C}_{6} \mathrm{H}_{5} \mathrm{OH}\right)$, uracil $\left(\mathrm{C}_{4} \mathrm{~N}_{2} \mathrm{O}_{2} \mathrm{H}_{4}\right)$, and the guanine-cytosine pair $\left(\mathrm{C}_{5} \mathrm{H}_{5} \mathrm{~N}_{5} \mathrm{O} \cdots \mathrm{C}_{4} \mathrm{H}_{5} \mathrm{~N}_{3} \mathrm{O}\right)$. For the largest molecule in the test set $\left(\left(\mathrm{C}_{4} \mathrm{H}_{9}\right)_{6} \mathrm{Li}_{6}\right)$ the number of independent atoms is 84 , if calculated without making use of the symmetry conditions. In all test cases the new algorithm

TABLE I. Number of iteration cycles for standard Hirshfeld-I method (std) and the new algorithms using Newton (H1), 2nd-order Householder (H2), 3rd-order Householder (H3), and polynomial (P) approach for obtaining of the fragment population $q_{A}$ for the second-row hydrides $\mathrm{AH}_{n}(\mathrm{~A}=\mathrm{Li}, \mathrm{Be}, \mathrm{B}, \mathrm{C}, \mathrm{N}, \mathrm{O}, \mathrm{F})$. An initial guess $x_{A}=0.5$

\begin{tabular}{|c|c|c|c|c|c|c|c|}
\hline & $\mathrm{LiH}$ & $\mathrm{BeH}_{2}$ & $\mathrm{BH}_{3}$ & $\mathrm{CH}_{4}$ & $\mathrm{NH}_{3}$ & $\mathrm{H}_{2} \mathrm{O}$ & $\mathrm{HF}$ \\
\hline std & 23 & 32 & 27 & 33 & 39 & 29 & 24 \\
\hline H1 & 4 & 5 & 3 & 3 & 4 & 4 & 3 \\
\hline $\mathrm{H} 2$ & 4 & 4 & 3 & 2 & 3 & 3 & 3 \\
\hline H3 & 4 & 4 & 3 & 2 & 3 & 3 & 3 \\
\hline $\mathrm{P}$ & 4 & 5 & 3 & 3 & 4 & 4 & 3 \\
\hline$q_{A}$ & 0.889 & 1.085 & 0.641 & -0.464 & -1.180 & -0.921 & -0.539 \\
\hline
\end{tabular}
(Eq. (6)) has been used in all the cases. 


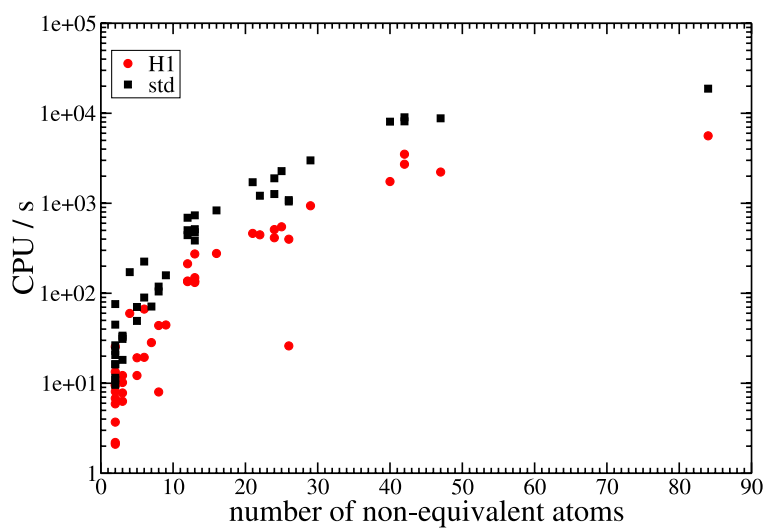

FIG. 1. CPU times required to achieve a convergence of $10^{-6}$ for the total charge of all non-equivalent atoms for a given molecule.

converges to the same solution as obtained from standard Hirshfeld-I approach. Fig. 1 compiles to CPU times needed for convergence obtained on a single Intel i5 CPU for the standard approach (std), data are shown in black, as well as for the new algorithm (H1), data are shown by red points. Note the logarithmic scale for the CPU times. Despite the fact that a single iteration for the $\mathrm{H} 1$ approach needs more computer time than a single iteration in the standard procedure, since the number of integrals that need to be calculated is larger in the $\mathrm{H} 1$ algorithm, the overall timing is much more favorable for the $\mathrm{H} 1$ approach, since the number of iterations needed for convergence is largely suppressed compared to the original scheme. For all calculated molecules, the $\mathrm{H} 1$ approach is about ten times faster than the original Hirshfeld procedure.

\section{E. Effective shell densities for the Hirshfeld-I partitioning scheme}

Usually, the atomic reference densities for the Hirshfeld-I partitioning scheme are stemming from self-consistent calculations. This causes several problems, when input densities for highly charged anions are needed, since no doubly charged anion is stable..$^{5}$ In addition, highly charged fragments have significant charge concentration far away from the nucleus, leading to undesirable artefacts in the Hirshfeld-I procedure. ${ }^{5}$ To circumvent those problems, several attempts have been taken in the direction of forcing the extra electrons to bind by using a finite basis, ${ }^{2}$ computing reference atoms in periodic boxes and neglecting a part of the electron density ${ }^{4}$ and scaling the shape function of neutral fragments. ${ }^{5}$

This study presents another way of constructing reference densities. Since the Hirshfeld-I partitioning scheme is based on local properties, the commonly used restriction that reference densities shall be obtained from self-consistent calculations (which is a purely energetic criterium) is released. Instead, the focus is set on proper description of local properties. Atomic reference densities shall fulfill the following criteria: describe the proper number of electrons in the system, display proper local behavior with respect to the atomic shell structure. ${ }^{18}$ Additionally, it would be desirable to have fragment densities of adjustable size in order to account for effects of the local environment the fragment is placed in. Since the energetic criterium is released, the fragment densities for any number of

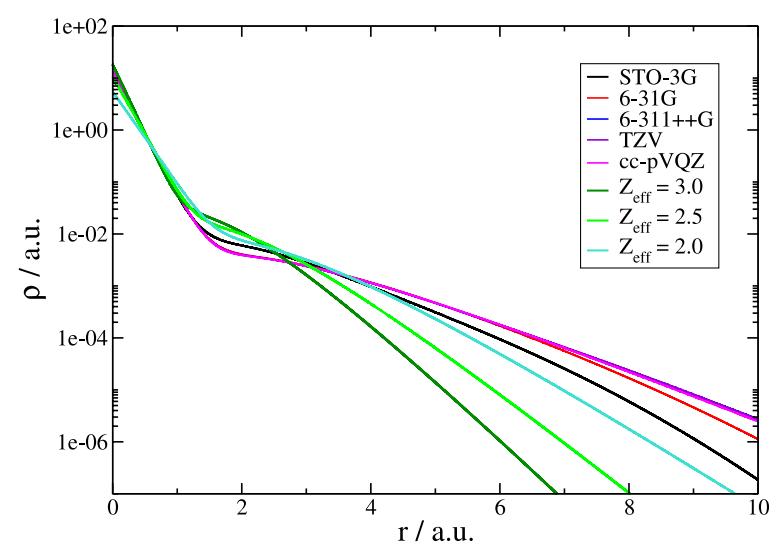

FIG. 2. Effective shell densities (ESDs) densities for the Li atom for different values of $Z_{\text {eff }}$ compared to atomic densities from standard basis sets.

electrons can simply be constructed as the spherical average over electron densities originating from a single Slater determinant, whereby the orbitals are given by the solutions of the one-electron Schrödinger-equation with $Z=Z_{\text {eff. }}$. Since those orbitals are stemming from a solution completely neglecting electron-electron repulsion, the resultant density will in general be compressed compared to a self-consistent density including this repulsion. That effect can be modeled by the parameter $Z_{\mathrm{eff}}{ }^{19,20}$ whereby $Z_{\mathrm{eff}}$ is the same for all orbitals in order to keep the scheme in its simplest form.

Figure 2 displays the new reference densities, hereinafter called effective shell densities (ESDs), for the $\mathrm{Li}$ atom for $Z_{\text {eff }}=3.0, Z_{\text {eff }}=2.5$, and $Z_{\text {eff }}=2.0$, respectively. All effective shell densities exhibit proper shell structure behavior, displaying a kink in the electron density as the boundary between the first shell and the second shell for the Li atom. For comparison, the figure also contains atomic densities using standard basis sets, showing that the electron density with $Z_{\mathrm{eff}}=3.0$ is largely compressed compared to a standard reference density. However, the size of the Li atom can be adjusted by the value of $Z_{\mathrm{eff}}$, as can be seen from Fig. 3, where the radius confining $99 \%$ of electron density of the Li atom is depicted (for comparison, $r_{99 \%}=6.27$ bohrs using STO-3G). Of course the Hirshfeld-I populations for the $\mathrm{Li}$ fragment in $\mathrm{LiH}$ depend on the chosen value of $Z_{\text {eff }}$, see the red colored data in Fig. 3. This

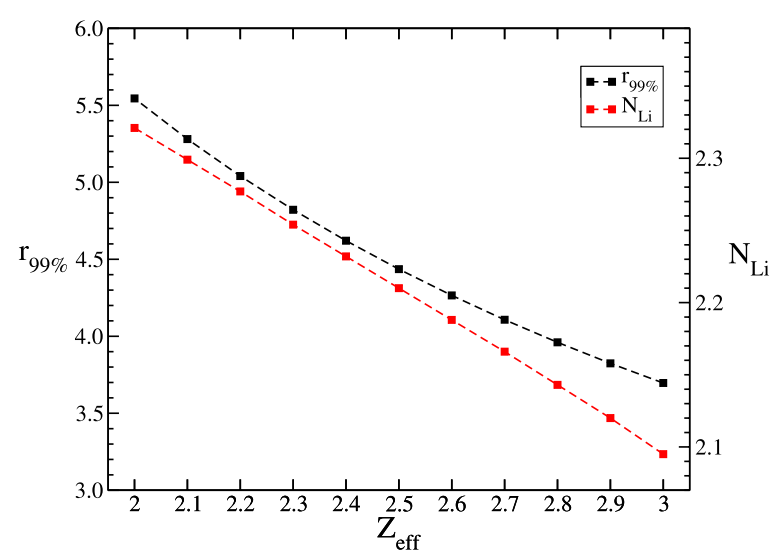

FIG. 3. Radius containing $99 \%$ of electron density of Li atom and Hirshfeld-I population of the Li fragment in $\mathrm{LiH}$ as a function of $Z_{\mathrm{eff}}$. 
TABLE II. Hirshfeld-I charge on oxygen $q_{\mathrm{O}}$ for water obtained with effective shell densities for different values of $Z_{\text {eff }}$.

\begin{tabular}{cc}
\hline \hline$Z_{\text {eff }}$ & $q_{\mathrm{O}}$ \\
\hline 5.2 & -0.274 \\
4.8 & -0.732 \\
4.4 & -1.221 \\
4.0 & -1.681 \\
3.6 & -1.967 \\
\hline \hline
\end{tabular}

effect is not specific for effective shell densities. In standard Hirshfeld-I calculations, the population of the Li fragment in $\mathrm{LiH}$ decreases from 2.59 electrons using STO-3G basis set to 2.04 electrons using a cc-pVQZ basis. But in the cases of Hirshfeld-I-ESD calculations, this effect is systematic and, therefore, could be exploited for tuning Hirshfeld-I charges, e.g., for accurately modeling, the electrostatic potential for force field calculations, an aim that was currently raised by Verstraelen et al., ${ }^{5}$ leading to the extended Hirshfeld method (Hirshfeld-E). Due to the construction of reference densities in the HE scheme (reference densities do more follow strict prescription of ensemble DFT in contrast to the Hirshfeld-I scheme $^{5}$ ) Hirshfeld-E and Hirshfeld-I are different methods, whereby Hirshfeld-I charges are more transferable than their Hirshfeld-E counterparts, since Hirshfeld-E charges take into account for the local environment in which the analyzed fragment is placed.

Please note the sensitivity of the Hirshfeld-I charges on the model parameter $Z_{\text {eff }}$, see Fig. 3 . This effect is more pronounced for increasing number of shells. For comparison, the Hirshfeld-I charges for water have been calculated based on the new atomic reference densities. Table II compiles the results. As can be seen from the data, the obtained charges vary from -0.3 electrons to -2.0 electrons for the range of $Z_{\text {eff }}$ between 5.2 and 3.6. For comparison, Hirshfeld-I calculation using standard basis sets yields a charge of -1.0 electrons on the oxygen side. The above example illustrates that the effective nuclear charge must be carefully chosen in order to correctly model the Hirshfeld-I charges. Another way to circumvent this ambiguity is to start from a given SCF solution for the neutral atom and simply add the remaining electrons via population of the virtual orbitals. The resulting densities also display proper local behavior, because the virtual orbitals introduce the atomic shell structure, since due to their nodal behavior.

Using effective shell densities as reference densities for the iterative Hirshfeld partitioning leads to a unified method, Hirshfeld-I-ESD providing either transferable charges by keeping $Z_{\text {eff }}$ fixed for a certain element or offering a more flexible scheme by allowing $Z_{\text {eff }}$ to adapt for the different local environments in which the corresponding fragment is placed.

\section{CONCLUSIONS}

It has been shown that atomic populations obtained from an iterative Hirshfeld (Hirshfeld-I) procedure can be interpreted as such populations which reproduce themselves. This requirement for the atomic population can be expressed analytically rather that being understood as the result of the orig- inal Hirshfeld-I procedure. Based on that analytical expression considerably faster algorithms for obtaining Hirshfeld-I charges have been developed. Usually convergence is reached within 4 steps, whereas more than 20 iterations are needed for the original Hirshfeld-I scheme.

In addition, new atomic reference densities for the Hirshfeld-I scheme have been proposed. The proposed ESDs are N-representable, exhibit proper shell structure, and can be obtained for any charged or uncharged species. ESD is obtained by spherical average over electron densities obtained from single determinants, whereby the orbitals are given by the solutions of one-electron Schrödinger-equation with effective nuclear charge $Z_{\text {eff. }}$ The resulting Hirshfeld-I-ESD scheme yields transferable charges in case that $Z_{\text {eff }}$ is fixed for given element or can be used as a flexible scheme with varying $Z_{\text {eff }}$ for different local environments, especially useful in force field applications.

\section{ACKNOWLEDGMENTS}

K.F. thanks the Alexander von Humboldt foundation for partially funding this work. A.M.P. and E.F. thank the spanish government, Grant No. CTQ2012-31174, for financial support.

\section{APPENDIX: GENERALIZATION OF THE NEW ITERATIVE ALGORITHM TO POLYATOMICS}

We show in this Appendix the generalization of the Newton method to obtain Hirshfeld-I atoms described in Section II B to an arbitrary molecule $\left[A_{a} B_{b} C_{c} \cdots\right]^{q}$, where the $a$ atoms of type $A$ have charge $q_{A}=Z_{A}-N_{A}$, the $b$ atoms of type $B$ have charge $\left(q_{B}=Z_{B}-N_{B}\right)$, etc. We represent the promolecular atomic density of an atom of type $R$ as in Eq. (5),

$$
\begin{array}{r}
\rho_{R_{k}}^{N_{R}}(\vec{r})=x_{R}\left[\rho_{R_{k}}^{l_{R}}(\vec{r})-\rho_{R_{k}}^{u_{R}}(\vec{r})\right]+\rho_{R_{k}}^{u_{R}}(\vec{r}) \\
\left(0 \leq x_{R} \leq 1\right), \quad(1 \leq k \leq r),
\end{array}
$$

where $R=A, B, \ldots, r=a, b, \ldots$, and $\rho_{R_{k}}^{N_{R}}(\vec{r})$ integrates to $u_{R}$ $-x_{R}$. The promolecular density is given by

$$
\rho^{0}(\vec{x} ; \vec{r})=\sum_{R} \sum_{k=1}^{r} \rho_{R_{k}}^{N_{R}(\vec{r})}
$$

where $\vec{x}=\left\{x_{A}, x_{B}, \ldots\right\}$ and the weight function for the $k$ th atom of type $R$ is

$$
w_{R_{k}}(\vec{x} ; \vec{r})=\rho_{R_{k}}^{N_{R}}(\vec{r}) / \rho^{0}(\vec{r}, \vec{x}) .
$$

The Hirshfeld-I atoms must fulfill the equations

$$
\begin{aligned}
& F_{1}(\vec{x})=\int w_{A_{i}}(\vec{x} ; \vec{r}) \rho(\vec{r}) d \vec{r}-u_{A}+x_{A}=0, \\
& F_{2}(\vec{x})=\int w_{B_{j}}(\vec{x} ; \vec{r}) \rho(\vec{r}) d \vec{r}-u_{B}+x_{B}=0,
\end{aligned}
$$

where $A_{i}, B_{j}, \ldots$ are, respectively, any of the atoms of type A, $\mathrm{B}, \ldots$ Using the matrix notation $\boldsymbol{F}(\vec{x}) \equiv\left[F_{1}(\vec{x}), \ldots, F_{N}(\vec{x})\right]^{T}$, the above linear system can be written as $\boldsymbol{F}(\vec{x})=\mathbf{0}$. The analogous to Eq. (18) is obtained by truncating at first order the Taylor expansion of $\boldsymbol{F}$ about a point $\vec{x}_{n}$, 


$$
\begin{aligned}
\boldsymbol{F}\left(\vec{x}_{n+1}\right) & \approx \boldsymbol{F}\left(\vec{x}_{n}\right)+\boldsymbol{J}\left(\vec{x}_{n}\right)\left(\vec{x}_{n+1}-\vec{x}_{n}\right)=\mathbf{0}, \\
\vec{x}_{n+1} & =\vec{x}_{n}-\boldsymbol{J}^{-1}\left(\vec{x}_{n}\right) \boldsymbol{F}\left(\vec{x}_{n}\right),
\end{aligned}
$$

or

$$
\Delta \vec{x}=-\boldsymbol{J}^{-1}\left(\vec{x}_{n}\right) \boldsymbol{F}\left(\vec{x}_{n}\right)
$$

where

$$
J_{R S}(\vec{x})=\left(\partial F_{R} / \partial x_{S}\right)=\int \frac{\partial}{\partial x_{S}} w_{R_{k}}(\vec{x} ; \vec{r}) \rho(\vec{r}) d \vec{r}+\delta_{R S}
$$

From Eqs. (A1)-(A3), we easily obtain

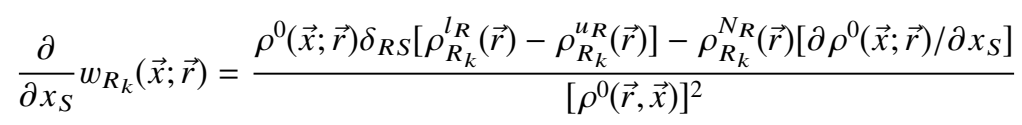

and

$$
\left[\partial \rho^{0}(\vec{x} ; \vec{r}) / \partial x_{S}\right]=\sum_{l=1}^{s}\left[\rho_{S_{l}}^{l_{S}}(\vec{r})-\rho_{S_{l}}^{u_{S}}(\vec{r})\right],
$$

where $s$ is the number of atoms of type $S$. Second and higher derivatives of $\rho^{0}(\vec{x} ; \vec{r})$ with respect to any of the variables contained in $\vec{x}$ are zero. As in the unidimensional case, Eq. (A7) or (A8) must be solved iteratively starting with a given input vector $\vec{x}_{0}$. More sophisticated iterative multidimensional methods, analogous to methods $\mathrm{H} 2$ and $\mathrm{H} 3$ of the unidimensional case can also be formulated. ${ }^{21}$ If there are $M$ types of atoms in the molecule, the dimension of $\boldsymbol{F}$ and $\boldsymbol{J}$ can be reduced to $N$ $=M-1$ and $(N \times N)$, respectively, since the equation $a q_{A}$ $+b q_{B}+\cdots=q$ should be satisfied. Instead, we can maintain the dimension $M$ when solving Eq. (A7) or (A8) and compare the final value of $a q_{A}+b q_{B}+\cdots$ with $q$ to test the results of the iterative process.

${ }^{1}$ F. L. Hirshfeld, Theor. Chim. Acta 44, 129 (1977).

${ }^{2}$ P. Bultinck, C. Van Alseneoy, P. W. Ayers, and R. Carbó-Dorca, J. Chem. Phys. 126, 144111 (2007).

${ }^{3}$ E. Francisco, A. Martín Pendás, A. Costales, and M. García-Revilla, Comput. Theor. Chem. 975, 2 (2011).

${ }^{4}$ D. E. P. Vanpoucke, P. Bultinck, and I. Van Driessche, J. Comput. Chem. 34, 405 (2013).
${ }^{5}$ T. Verstraelen, P. W. Ayers, V. Van Speybroeck, and M. Waroquier, J. Chem. Theory Comput. 9, 2221 (2013).

${ }^{6}$ P. Bultinck, P. W. Ayers, S. Fias, K. Tiels, and C. Van Alseneoy, J. Chem. Phys. 444, 205 (2007).

${ }^{7}$ E. R. Davidson and S. Chakravorty, Theor. Chim. Acta 83, 319 (1992).

${ }^{8}$ C. Fonseca Guerra, E. J. Handgraaf, J.-W. Baerends, and F. M. Bickelhaupt, J. Comput. Chem. 25, 189 (2004).

${ }^{9}$ R. F. Nalewajski and R. Loska, Theor. Chem. Acc. 105, 374 (2001)

${ }^{10}$ E. Francisco, A. Martín Pendás, M. A. Blanco, and A. Costales, J. Phys. Chem. A 111, 12146 (2007).

${ }^{11}$ G. R. Parr and W. Yang, Density-Functional Theory of Atoms and Molecules (Oxford University Press, 1989).

${ }^{12}$ E. Francisco, A. Martín Pendás, and M. A. Blanco, J. Chem. Phys. 126, 094102 (2007).

${ }^{13}$ A. Martín Pendás, E. Francisco, and M. A. Blanco, J. Chem. Phys. 127, 144103 (2007)

${ }^{14}$ E. Francisco and A. Martín Pendás, Comput. Phys. Commun. 185, 2663 (2014).

${ }^{15}$ W. M. Schmidt, K. K. Baldridge, J. A. Boatz, S. T. Elbert, M. S. Gordon, J. H. Jensen, S. Koseki, N. Matsunaga, K. A. Nguyen, S. J. Su et al., J. Chem. Phys. 14, 1347 (1993).

${ }^{16}$ The PROMOLDEN QTAIM/IQA code is available from the authors (A Martín Pendás and E. Francisco) upon request.

${ }^{17}$ A. Martín Pendás, M. A. Blanco, and E. Francisco, J. Chem. Phys. 120, 4581 (2004).

${ }^{18}$ K. Finzel, Int. J. Quantum Chem. 114, 1546 (2014).

${ }^{19}$ J. C. Slater, Phys. Rev. 36, 57 (1930).

${ }^{20}$ C. Zener, Phys. Rev. 36, 51 (1930).

${ }^{21}$ M. A. Hafiz and M. S. M. Bahgat, J. Math. Comput. Sci. 2, 1200 (2012). 